\title{
28 Research Soure \\ Knee Joint Rotation Axis Is More Laterally Rotated and Valgus Than We Previously Thought
}

\section{Liwen Zheng ( $\square$ zhengliwen@csu.edu.cn )}

Department of Orthopedics, The Second Xiangya Hospital, Central South University. No.139, middle renmin road, Changsha, Hunan, China.

\section{Deye Song}

Second Xiangya Hospital of Central South University

\section{Yongheng Luo}

Second Xiangya Hospital of Central South University

\section{Wanchun Wang}

Second Xiangya Hospital of Central South University

\section{Xinzhan Mao}

Second Xiangya Hospital of Central South University

\section{Lei Wang}

Central South University

\section{Research Article}

Keywords: Rotation axis of knee joint, Stereogrammetry, TKA

Posted Date: January 7th, 2021

DOl: https://doi.org/10.21203/rs.3.rs-138968/v1

License: (c) (1) This work is licensed under a Creative Commons Attribution 4.0 International License.

Read Full License 


\section{Abstract}

Background: Currently, the patient satisfaction rate after receiving TKA is around $80 \%$ to $90 \%$ and there is still room for further improvement. With the aim to provide reference for improving TKA surgery, stereophotogrammetry was used in this study to evaluate knee joint kinematics.

Methods: Multiple MRI images of knee joints flexed at $0,10,20 \ldots$ to 100 degrees were scanned in bilateral knees of 45 asymptomatic adult volunteers. After three-dimensional alignment of the femur, the displacement and rotation angles of the tibia flexed at different angles were measured. Contributing factors were tested using One-way ANOVA and chi-square test in SPSS 24.

Results: 1)No contributing factors was found to influence rotation axis; 2) Within the range of motion at $0-100^{\circ}$ of flexion, the rotation axis exhibited no significant displacement relative to the femur. 3 ) On the horizontal plane, the lateral rotation angle of knee joint axis was $4.91^{\circ} \pm 1.84^{\circ}\left(3^{\circ} \sim 7^{\circ}\right)$ relative to transepicondylar axis (TEA) and $7.84^{\circ} \pm 2.09^{\circ}$ relative to posterior condylar axis (PCA); on the coronal plane, the valgus angle of knee joint axis was $3.38^{\circ} \pm 0.82^{\circ}\left(2.5^{\circ} \sim 5^{\circ}\right)$ relative to tibial plateau, and $6.53^{\circ}$ $\pm 0.91^{\circ}\left(5^{\circ} \sim 8^{\circ}\right)$ relative to the perpendicular line of the lower limb force line.

Conclusion: No contributing factors were found, but the rotation axis of the knee joint is more laterally rotated and valgus than previously thought. These findings may explain the research result of some current literatures, and may provide theoretical rationale for further improvement of knee prosthesis design.

\section{Introduction}

Total knee arthroplasty (TKA) has been proved to be a successful surgery and it can substantially improve the quality of life in patients with end-stage knee joint diseases. The patient satisfaction rate has been improved from $81 \%$ to $85 \%$ from 1990 to 2012[23], at a relatively moderate speed. Currently, the satisfaction rate among patients receiving TKA is around $80 \%$ to $90 \%[5,15,23]$, but there is still room for further improvement. In recent decades, numerous studies have been carried out to explore knee joint kinematics with the aim to improve the related patient satisfaction rate, and the knee joint rotation axis was one of the subjects of these investigations. It has been generally believed in academic circles that the position of the knee joint rotation axis relative to the femur shifts with the change of the flexion angle of the knee joint, and the motion trajectory is J-shaped $[2,8,12,18]$. In addition, the concept of femoral rollback has been widely recognized for a long time and is commonly described in current literature reports $[7,11,14,25,27]$.

To find clues for further modification of TKA related design, our study team developed a stereophotogrammetry technique that combined MRI imaging and programming technique for conducting in vivo studies of knee joints in healthy individuals. Our study results better described the true kinematics of knee joints than the above two conclusions, thus they may provide theoretical rationale for further improvement in knee joint surgical techniques and prosthesis design. 


\section{Materials And Methods}

\section{Data collection and Statistical analysis}

From January 2020 to November 2020, MRI imaging data of bilateral knee joints was collected from 45 healthy adult volunteers. This study was reviewed and approved by the Ethics Committee of the hospital, and all volunteers participating in the study signed the informed consent.

All the volunteers were scanned in a supine position using the Siemens Skyra 3.0T MRI system. Each volunteer was first required to straighten one of his or her knee joints to have the full-length of the lower limb scanned from the femoral head to the ankle joint. The voxel element was a cube with a side length of $3 \mathrm{~mm}$, and the volume data was labeled as VLMtemp3. The volunteers were then asked to flex their knees at angles ranging from $0,10,20$...to 100 degrees, respectively, so as to specifically scan the knee joints respectively. The voxel element was a cube with a side length of $0.5 \mathrm{~mm}$, and the volume data were labeled as VLM0, VLM10, VLM20... to VLM100. All the volume data were exported from the console in the format of DICOM file.

For length measurements, the distance from the center of the femoral head to the center of the knee was defifined as the femoral length. The total limb length was defined as the distance between the center of the femoral head to the center of the ankle.

One-way ANOVA was used to analyze the correlation between knee joint rotation axis and BMI, age, femur length, and total length of lower limbs, and chi-square test was used to test the correlation between knee joint rotation axis and gender. $\mathrm{P}<0.05$ were considered significant. We used SPSS version 24 for all analyses.

\section{The three-dimensional position and rotation of tibia relative to femur during knee joint flexion were measured}

Herein, the concept of central alignment needs to be first explained, as it will be frequently mentioned in the sections below. If an object is centrally aligned in volume VLM1 and volume VLM2, it means that this object in VLM1 and VLM2 will be completely overlapped if the volume center of VLM1 is overlapped with that of VLM2.

All measurement operations were conducted by using Matlab software; please refer to the appendix for measurement methods.

In order to reduce the workload, most of the measurement operations were automatically completed by the computer based on the in-house codes written in advance.

As the computer could not automatically distinguish the respective MRI data representing the femur, tibia and soft tissue, the central alignment of tibia/femur in the volume data from the same volunteer scanned at different knee flexion angles had to be completed manually. In order to maximize the accuracy of the 
alignment, the following measures were implemented in this study: 1 ) a position resolution of $0.5 \mathrm{~mm}$ was adopted to ensure optimally high definition MRI images; 2) When a certain volume of VLM1 was rotated to ensure the tibia in VLM1 and VLM2 have the same three-dimensional orientation, two slices with marked features of tibia were initially explored among the images taken on the coronal plane (perpendicular to the X-axis) in VLM1; if another two slices with the same features of tibia could be identified in VLM2, meanwhile the inter-plane spaces of the two volume were equivalent, it would indicate that the direction of tibia has been aligned in the X-axis images. Alignment of the direction of tibia in the $\mathrm{Y}$-axis (perpendicular to the sagittal plane) images and Z-axis (perpendicular to the horizontal plane) images was also performed in the same manner, thus precise consistency between the three-dimensional orientation of the tibia of VLM1 and VLM2 could be ensured (Figure 1). this method is also used to align femur;3) After the direction of tibia was aligned in VLM1 and VLM2, a distinctive slice was identified among VLM1 X-axis images and its counterpart plane was found in VLM2 and the difference in the sequence numbers of the two slices would represent the relative position of tibia in VLM1 and VLM2 in Xaxis direction. Similarly, the relative positions of tibia in VLM1 and VLM2 in Y-and Z-axis directions could be determined. This method is also used to determine the relative position of the femur in VLM1 and VLM2. 4) As to the location of FPtibia2 (Figure 2 and the appendices) in VLM90_Femur, FPtibia2 was initially precisely located in VLM0_Tibia, and then the coordinate of FPtibia2 in VLM90_Femur was calculated based on the parameters of VLM90_Tibia_transfer_Femur. Note: FPtibia2 in VLM90_Femur should not be manually positioned as this manipulation is subjected human error due to variations in tibial direction in VLM90_Femur and VLM0_Tibia.

\section{Results}

Relevant data was measured in bilateral knees of 45 asymptomatic adult volunteers, the characteristics of them was summarized in Table 1.

If the femur is centrally aligned in VLMX_Tibia $(X=10,20 \ldots 100)$ and VLM0_Tibia, the trajectories of different feature points of tibia is shown in the figure above (Figure 2). Rotate VLM0_Tibia so that the planes on which the trajectories of all feature points locate are parallel to the sagittal plane (Figure 3 ), and seen from the sagittal plane, the movements of all the feature

points form an approximately circular motion. It can be seen that after VLMO_Tibia is rotated from the direction displayed in Figure 2 to that in Figure 3, the rotation axis of all feature points is parallel to the Yaxis. Based on the rotation parameters, the direction of the rotation axis in Figure 2 (VLM0_Tibia) can be inferred. In this study, the relationship between the direction of rotation axis and the references used to determine the direction of the femoral prosthesis during TKA is shown in Table 2.

Taking BMI, age, femur length, and lower limb length as dependent variables, analyze their influence on various parameters of the rotation axis, and the results are shown in Table 3.

\section{Discussion}


This study did not find any factors influencing the direction of the rotation axis, but surprisingly, the results showed that the rotation axis was more laterally rotated and valgus than generally thought. In addition to the measurement results presented in Table 2, another more intuitive evidence was provided to prove that the rotation axis is not parallel to TEA (Figure 4). Most of the previous studies that explored how to align femoral prosthesis horizontally had tacitly accepted the hypothesis that TEA was parallel to the rotation axis[1,4,6]. Although there have been a few studies exploring the relationship between rotation axis and TEA, this hypothesis has not been explicitly denied due to the limit in the precision of the measurement methods,[19]; whereas in this study, imaging evidence was provided for the first time to deny this hypothesis in an intuitive way(Figure 4), moreover, the angle between the rotation axis and TEA was quantitatively measured (Table 2 ).

During knee joint flexion and extension, the tibia may undergo mild degrees of inversion/eversion and internal/external rotation. It was observed that varus/valgus and internal/external rotation caused most of the feature points to swing around the circular arc formed by the rotational motions (Figure 2 and Figure 3). But after temporal swinging, these points would quickly return to the circular arc to restore the rotation axis--no continuous motions would be made in any certain direction, and the radius of rotation would not be continuously reduced/increased. Our results showed that little-to-no changes occurred to the rotation axis when the knee joint was flexed within the range of 0-100 degrees and that the femoral rollback was not readily noticeable or did not exist at all within this range of motion, although very limited supporting evidence could be identified from the literature[22].

The main reason made this result seemingly counterintuitive was that the shapes of the medial and lateral condyles of the femur on sagittal plane were not circular, so it is hard to imagine how the tibia moves in a circular motion relative to the femur. Further investigations were made in this study to verify the experimental results. It was revealed that the motion of the knee joint was quite complex (Figure 5), so the impacts of the sagittal plane shape of the medial and lateral condyles of the femur on the motion of the knee joint should not be overestimated. Therefore, objective results could not be yielded based on the methodology of inferring the motion of rotation axis via accurately measuring the shape of the medial and lateral condyles of the femur on the sagittal plane, although this method has been used in some recently published studies $[18,20,21,22]$. Medial displacement of tibia during knee flexing (Figure 4) may partially accounts for the lateral displacement of the tibiofemoral joint contact area. In this study, we found that the size of the joint space and joint contact area changed in a subtle and complicated way with the increase of knee flexing angle. This phenomenon, may explain the paradox that the shape of the sagittal planes of the medial and lateral femoral condyles is not circular while tibia moves in circular motion relative to the femur --the shape of femoral condyles on the sagittal plane is not a crucial factor.

Based on the above discussion, it can be concluded that the femoral prosthesis shall meet the following requirements to reproduce the tibial motion pattern of a healthy knee within the range of motion of $0-100$ degree: 1) its rotation axis has been fixed to preclude rollback; 2)its rotation axis is everted at $6.53^{\circ} \pm 0.91^{\circ}$ relative to the perpendicular line of the lower limb force line, externally rotated at $7.84^{\circ} \pm 2.09$ relative to the PCA; In comparison, the currently canonical TKA surgical technique (Campbell's Operative Orthopedics, 
13th Edition) adopts the following criteria for implanting femoral prostheses: the rotation axis of the femoral prosthesis is perpendicular to the lower limb force line on the coronal plane and parallel to TEA on the horizontal plane. Obviously, the current surgical technique may result in changes in the rotation axis of the knee joint after TKA, that is, approximately $6^{\circ}$ of varus on the coronal plane and $5^{\circ}$ of internal rotation on the horizontal plane)(Table 2).

A literature retrieval has been conducted among studies published in recent years on the correlation between internal/external rotation of femoral prosthesis and patient satisfaction rate. Many researchers believe that internal rotation of femoral prosthesis has greater negative impacts on the surgery and during the surgery, a slight angle of external rotation is preferred so as to avoid internal rotation $[3,9,10,13,26]$. On the contrary, no articles had been identified that suggested greater negative effects of external rotation of femoral prosthesis. This phenomenon could be explained by the result of this study, that is, the rotation axis was already internally rotated at approximately $5^{\circ}$ when the femoral prosthesis was implanted in parallel to the TEA on the horizontal plane using the current surgical technique; if the internal rotation angle of the femoral component was further increased, the changes in the direction of the rotation axis after the surgery would be enhanced, which would increase the risks of postsurgical complications and decrease patient satisfaction rate. If the femoral prosthesis was implanted at a slight increased angle of external rotation, the changes in the direction of the rotation axis after the surgery would be minimized so as to improve the surgical efficacy. In addition, our results suggest that the radius of rotation of the lateral femoral condyle should be designed to be larger than that of the medial condyle because this may restore the valgus of the rotation axis in the coronal plane while avoiding excessive valgus of the articular surface. Nevertheless, the trochlear position in the femoral prosthesis should also be adjusted accordingly, which warrants further studies in the future.

In this study, the motion of tibia relative to the femur was quantitatively measured, and the following conclusions were drawn :1) The rotation axis has no significant displacement relative to the femur when the knee flexion angle was kept at least within the range of 0-100 degree; 2) the currently prevailing surgical technique for TKA and the correspondingly designed knee prosthesis may cause varus and internal rotation of the rotation axis; 3 ) the method of extrapolating the position of the axis of rotation through accurately measuring the sagittal plane shape of the medial and lateral condyles of the femur has yielded no accurate results.

\section{Declarations}

-Ethics approval and consent to participate: This study was reviewed and approved by the Clinical Research Ethics Committee of the Second Xiangya Hospital of Central South University, and all volunteers participating in the study signed the informed consent.

-All methods in the study were carried out in accordance with the Helsinki guidelines and declaration.

-Consent for publication: Not applicable. 
-Availability of data and materials: Available as related files

-Competing interests: There is no conflict of interest.

-Funding: Not applicable

-Authors' contributions

Deye Song contributed to the conception of the study;

Deye Song and Liwen Zheng performed the experiment;

Wan-Chun Wang contributed significantly to analysis and manuscript preparation;

Liwen Zheng and Xinzhan Mao performed the data analyses and wrote the manuscript;

Yongheng Luo and Lei Wang helped perform the analysis with constructive discussions.

-Acknowledgements: Not applicable

\section{References}

1.Akagi M, Yamashita E, Nakagawa T, Asano T, Nakamura T. Relationship between frontal knee alignment and reference axes in the distal femur. Clin Orthop Relat Res, 2001;(388):147-56.

2.Anne MH, Sanjay J, Anoop KS, William WS, Anrei GL. The axes of rotatin of the knee. Clin Orthop Relat Res; (1993); 290:259-258.

3.Bhattee G, Moonot P, Govindaswamy R, Pope A, Fiddian N, Harvey A. Does malrotation of components correlate with patient dissatisfaction following secondary patellar resurfacing? Knee,2014;21(1):247-51.

4.Blakene W, Beaulieu Y, Puliero B, Kiss MO, Vendittoli PA. Bone resection for mechanically aligned total knee arthroplasty creates frequent gap modifications and imbalances. Knee Surg Sports Traumatol Arthrosc, 2020;28(5):1532-1541.

5.Bourne RB, Chesworth BM, Davis AM, Mahomed NN, Charron KD. Patient satisfaction after total knee arthroplasty: who is satisfied and who is not? Clin Orthop Relat Res, 2010;468(1):57-63.

6.David WF, Daniel JJ, Matthew JH, Ryan S, Tyler JK, David WM. Relationship of the Posterior Condylar Line and the Transepicondylar Axis: A CT-Based Evaluation. J Knee Surg, 2020;33(7):673-677.

7.Iriuchishima T, Ryu. A Comparison of Rollback Ratio Between Bicruciate Substituting Total Knee Arthroplasty and Oxford Unicompartmental Knee Arthroplasty. J Knee Surg, 2018;31(6):568-572.

8.Iwaki H, Pinskerova V, Freeman MAR. Tibiofemoral movement 1: the shapes and relative movements of the femur and tibia in the unloaded cadaver knee. J Bone Joint Surg [Br], 2000; 82-B(8):1189-1195. 
9.Joseph L, Batailler C, Roger J, Swan J, Servien E, Lustig S. Patellar component size effects patellar tilt in total knee arthroplasty with patellar resurfacing. Knee Surg, 2020. doi: 10.1007/s00167-020-05984-9.

10.Kawahara S, Okazaki K, Matsuda S, Nakahara H, Okamoto S, Iwamoto Y. Internal rotation of femoral component affects functional activities after TKA-survey with the 2011 Knee Society Score. J Arthroplasty, 2014;29(12):2319-23.

11.Kim H, Pelker RR, Gibson DH, Irving JF, Lynch JK. Rollback in Posterior Cruciate Ligament-Retaining Total Knee Arthroplasty. A Radiographic Analysis. J Arthroplasty, 1997;12(5):553-61.

12.Kim K, Feng J, Nha KW, Park WM and Kim YH. Improvement of the knee center of rotation during walking after opening wedge high tibial osteotomy. J Engin Med,2015, 229(6) 464-468.

13.Koh DTS, Woo YL, Yew AKS, Yeo SJ. Kinematic aligned femoral rotation leads to greater patella tilt but similar clinical outcomes when compared to traditional femoral component rotation in total knee arthroplasty. A propensity score matched study. Knee Surg Sports Traumatol Arthrosc, 2020. doi:

10.1007/s00167-020-06081-7.

14.Koh IJ, Lin CC, Patel NA, Chalmers CE, Maniglio M, Han SB, McGarry MH and Lee TQ. Kinematically Aligned Total Knee Arthroplasty Reproduces More Native Rollback and Laxity Than Mechanically Aligned Total Knee Arthroplasty: A Matched Pair Cadaveric Study. Orthop Traumatol Surg Res, 2019;105(4):605611.

15.Malou EM, José MH, Petra JC, Cornelia HM. Definitions of poor outcome after total knee arthroplasty: an inventory review. BMC Musculosk Disord, 2020; 21:378.

16.Murray DW, Parkinson RW. Usage of unicompartmental knee arthroplasty. J Bone Joint Surg [Br], 2018;100-B:432-435.

17.Niccoli G, Benedetto PD, Salviato D, Beltrame A, Gisonni R, Cainero V, Causero A. Can UKA after KineSpring system failure be a viable option? A case report. Acta Biomed, 2019; 90(1): 192-197.

18.Okamoto S, Mizu-uchi H, Okazaki K, Hamai S, Tashiro Y, Nakahara H, Iwamoto Y. Two-dimensional planning can result in internal rotation of the femoral component in total knee arthroplasty. Knee Surg Sports Traumatol Arthrosc, 2016; 24:229-235.

19. Oussedik S, Scholes C, Ferguson D, Roe J, Parker D. Is Femoral Component Rotation in a TKA Reliably Guided by the Functional Flexion Axis? Clin Orthop Relat Res, 2012; 470:3227-3232.

20.Park A, Duncan ST, Nunley RM, Keeney JA, Barrack RL, Nam D. Relationship of the Posterior Femoral Axis of the Kinematically Aligned Total Knee Arthroplasty to the Posterior Condylar, Transepicondylar, and Anteroposterior Femoral Axes. Knee. 2014;21(6):1120-1123. 
21.Pate VV, Hall K, Ries M, Lotz J, Ozhinsky E, Lindsey C, Lu Y Majumdar S. A three-dimensional MRI analysis of knee kinematics. J Orthop Res, 2004;(22):283-292.

22.Pinskerova V, Johal P, Nakagawa S, Sosna A, Williams A, Gedroyc W, Freeman MAR. Does the femur roll-back with flexion? J Bone Joint Surg [Br], (2004)86-B:925-31.

23.Schulze A, Scharf HP. Satisfaction after total knee arthroplasty. Comparison of 1990-1999 with 20002012. Orthopade, 2013;42(10):858-65.

24.Vecchini E, Ditta A, Gelmini M, Maluta T, Valentini R, Ricci M, Magnan B. Rupture of the femoral component and severe metallosis of the knee 10 years after unicompartmental knee arthroplasty (UKA): a case report. Acta Biomed, 2019; 90(1): 198-202.

25.Wachowski MM, Walde TA, Balcarek P, Schüttrumpf JP, Frosch S, Stauffenberg C, Frosch KH, Fiedler C, Fanghänel J, Meesenburg DK and Nägerl H. Total Knee Replacement With Natural Rollback. Ann Anat, 2012;194(2):195-9.

26.Woiczinski M, Kistler M, Schröder C, Braun C, Weber P, Müller PE, Jansson V, Steinbrück A. TKA designintegrated trochlea groove rotation reduces patellofemoral pressure. Knee Surg, (2019) 27:1680-1692.

27.Zuppinger H. Die aktive flexion im unbelasteten Kniegelenk:Züricher Habil. Schr. Wiesbaden: Bergmann, 1904:703-63.

\section{Tables}

Table 1 Subject characteristics

\begin{tabular}{|llll|}
\hline Gender & Male & Female & Both \\
\hline Number of volunteers & $27 / 45(60.0 \%)$ & $18 / 45(40.0 \%)$ & $45(100.0 \%)$ \\
\hline Age $(\mathrm{yrs})$ & $49.4 \pm 16.1$ & $45.6 \pm 19.3$ & $47.9 \pm 17.5$ \\
\hline BMl & $24.6 \pm 1.59$ & $22.1 \pm 1.86$ & $23.6 \pm 2.1$ \\
\hline Femur length $(\mathrm{cm})$ & $52.9 \pm 3.43$ & $48.3 \pm 3.88$ & $51.0 \pm 4.26$ \\
\hline Lower limb length $(\mathrm{cm})$ & $89.3 \pm 6.58$ & $83.8 \pm 3.82$ & $87.1 \pm 6.24$ \\
\hline
\end{tabular}

Table 2 Relationship between rotation axis direction and femoral prosthesis references during TKA 


\begin{tabular}{|c|c|c|c|c|}
\hline $\begin{array}{l}\text { On the horizontal } \\
\text { plane }\end{array}$ & Male(54 knees) & Female(36 knees) & Both(90 knees) & $\begin{array}{l}\mathrm{P} \\
\text { value(between } \\
\text { genders) }\end{array}$ \\
\hline TEA & $\begin{array}{l}4.88^{\circ} \\
\pm 1.86^{\circ}\left(3^{\circ} \sim 7^{\circ}\right)^{*}\end{array}$ & $\begin{array}{l}4.96^{\circ} \\
\pm 1.76^{\circ}\left(3^{\circ} \sim 7^{\circ}\right)^{\star}\end{array}$ & $\begin{array}{l}4.91^{\circ} \\
\pm 1.84^{\circ}\left(3^{\circ} \sim 7^{\circ}\right)^{*}\end{array}$ & 0.816 \\
\hline PCA & $\begin{array}{l}7.78^{\circ} \\
\pm 2.17^{\circ}\left(5^{\circ} \sim 12^{\circ}\right)^{\star}\end{array}$ & $\begin{array}{l}7.94^{\circ} \\
\pm 1.9 .6^{\circ}\left(5^{\circ} \sim 12^{\circ}\right)^{\star}\end{array}$ & $\begin{array}{l}7.84^{\circ} \\
\pm 2.09^{\circ}\left(5^{\circ} \sim 12^{\circ}\right)^{*}\end{array}$ & 0.385 \\
\hline \multicolumn{5}{|l|}{ On the coronal plane } \\
\hline $\begin{array}{l}\text { Perpendicular line of } \\
\text { the lower limb force } \\
\text { line }\end{array}$ & $\begin{array}{l}6.52^{\circ} \\
\pm 0.96^{\circ}\left(5^{\circ} \sim 8^{\circ}\right) \star \star\end{array}$ & $\begin{array}{l}6.56^{\circ} \\
\pm 0.83^{\circ}\left(5^{\circ} \sim 8^{\circ}\right)^{\star \star}\end{array}$ & $\begin{array}{l}6.53^{\circ} \\
\pm 0.91^{\circ}\left(5^{\circ} \sim 8^{\circ}\right)^{\star \star}\end{array}$ & 0.753 \\
\hline Tibial plateau & $\begin{array}{l}3.37^{\circ} \\
\pm 0.89^{\circ}\left(2^{\circ} \sim 5^{\circ}\right)^{\star \star}\end{array}$ & $\begin{array}{l}3.39^{\circ} \\
\pm 0.70^{\circ}\left(2.5^{\circ} \sim 4.5^{\circ}\right)^{\star \star}\end{array}$ & $\begin{array}{l}3.38^{\circ} \\
\pm 0.82^{\circ}\left(2^{\circ} \sim 5^{\circ}\right)^{\star \star}\end{array}$ & 0.571 \\
\hline
\end{tabular}

\section{Table $3 \mathrm{P}$ values between parameters of rotation axis and contributing factors}

\begin{tabular}{|c|c|c|c|c|}
\hline On the horizontal plane & $\mathrm{BMI}$ & Age & $\begin{array}{l}\text { Femur } \\
\text { length }\end{array}$ & $\begin{array}{l}\text { Lower limb } \\
\text { length }\end{array}$ \\
\hline Angle with TEA & 0.904 & 0.792 & 0.300 & 0.645 \\
\hline Angle with PCA & 0.192 & 0.151 & 0.725 & 0.505 \\
\hline \multicolumn{5}{|l|}{ On the coronal plane } \\
\hline $\begin{array}{l}\text { Angle with the perpendicular line of the lower limb } \\
\text { force line }\end{array}$ & 0.505 & 0.216 & 0.995 & 0.663 \\
\hline Angle with tibial plateau & 0.105 & 0.582 & 0.281 & 0.162 \\
\hline
\end{tabular}

\section{Figures}



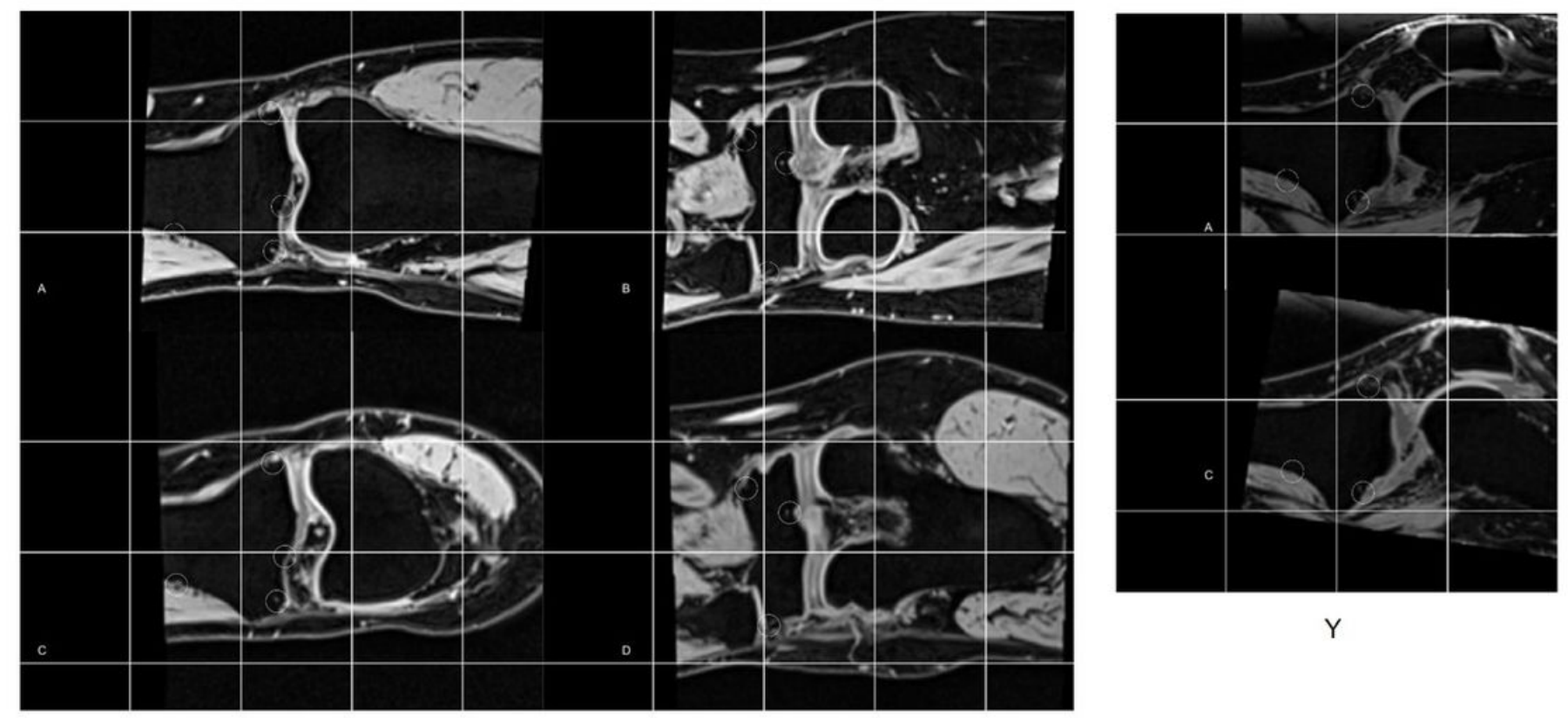

X

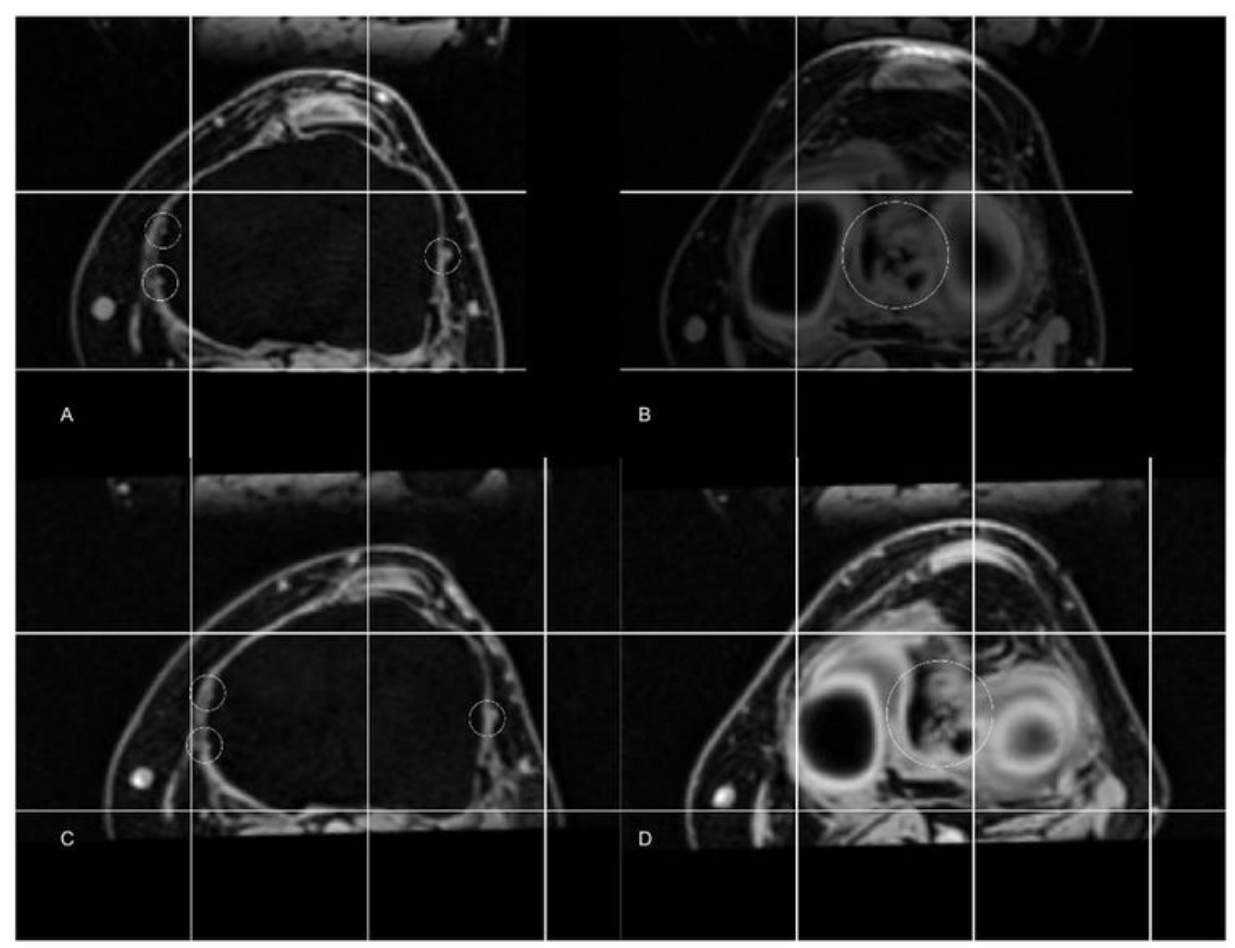

z

\section{Figure 1}

1X-Z In MRI images of knee joints, the $X, Y$ and Z-axis are perpendicular to the coronal, sagittal and horizontal planes, respectively. The circles mark the feature areas of the tibia, indicating that the slices of $A$ and $C$ of the tibia are the same, and that of $B$ and $D$ are the same. And the slice interval between $A$ and $B$ is equal to that between $C$ and D. A) Slice of VLM0_Tibia B) Slice of VLM0_Tibia C) Slice of VLM50_Tibia D) Slice of VLM50_Tibia 


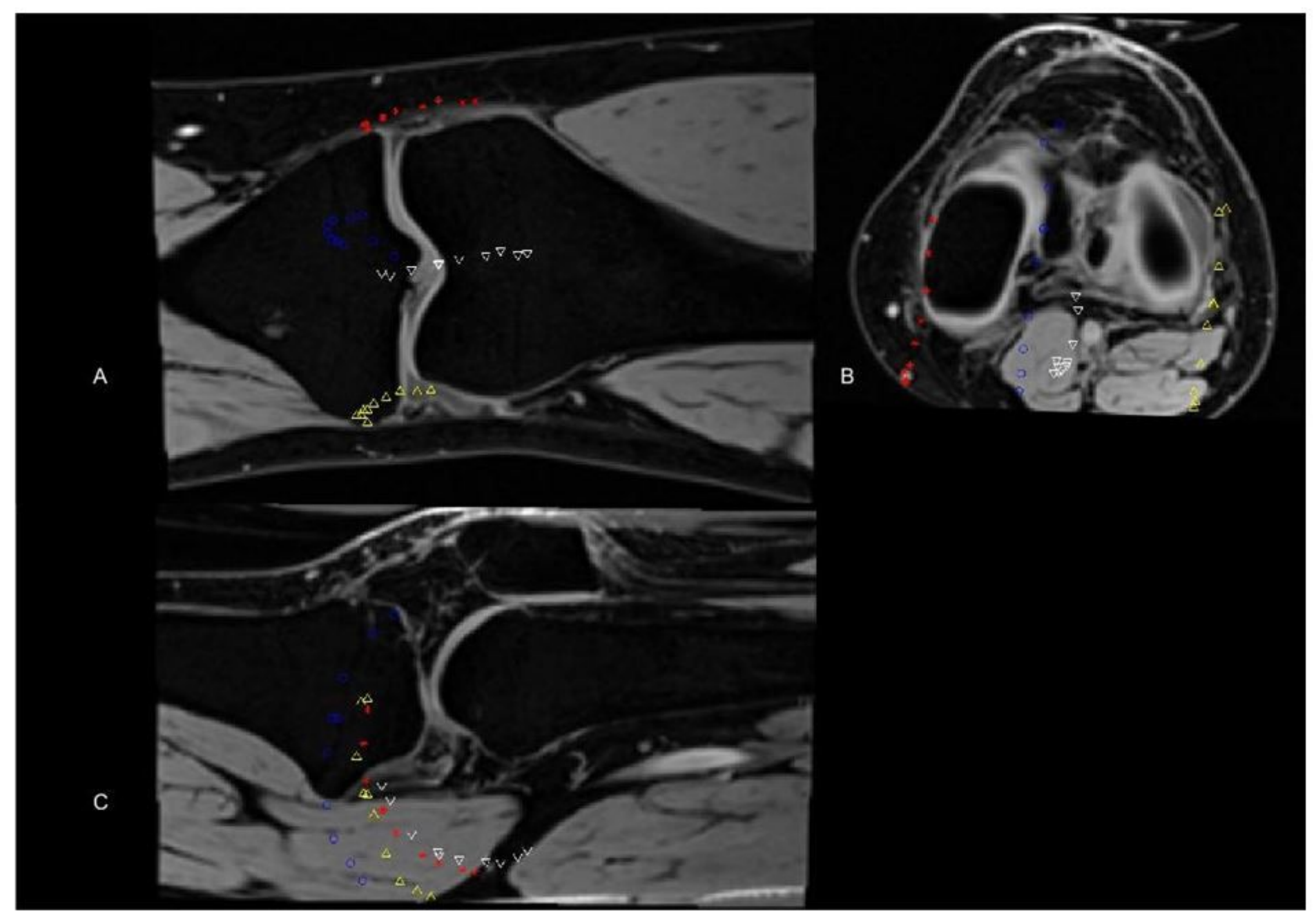

Figure 2

In this study, the VLM0_Tibia was employed as the reference to observe the positions of several feature points relative to the femur. Inverted white triangle: FPtibia1, tibial insertion of posterior cruciate ligament (PCL) Blue circle: FPtibia2, located in the middle of the anterior margin of tibial plateau Yellow triangle: FPtibia3, located in the middle of the lateral margin of tibial plateau Red asterisk: FPtibia4, located in the middle of the medial margin of tibial plateau 


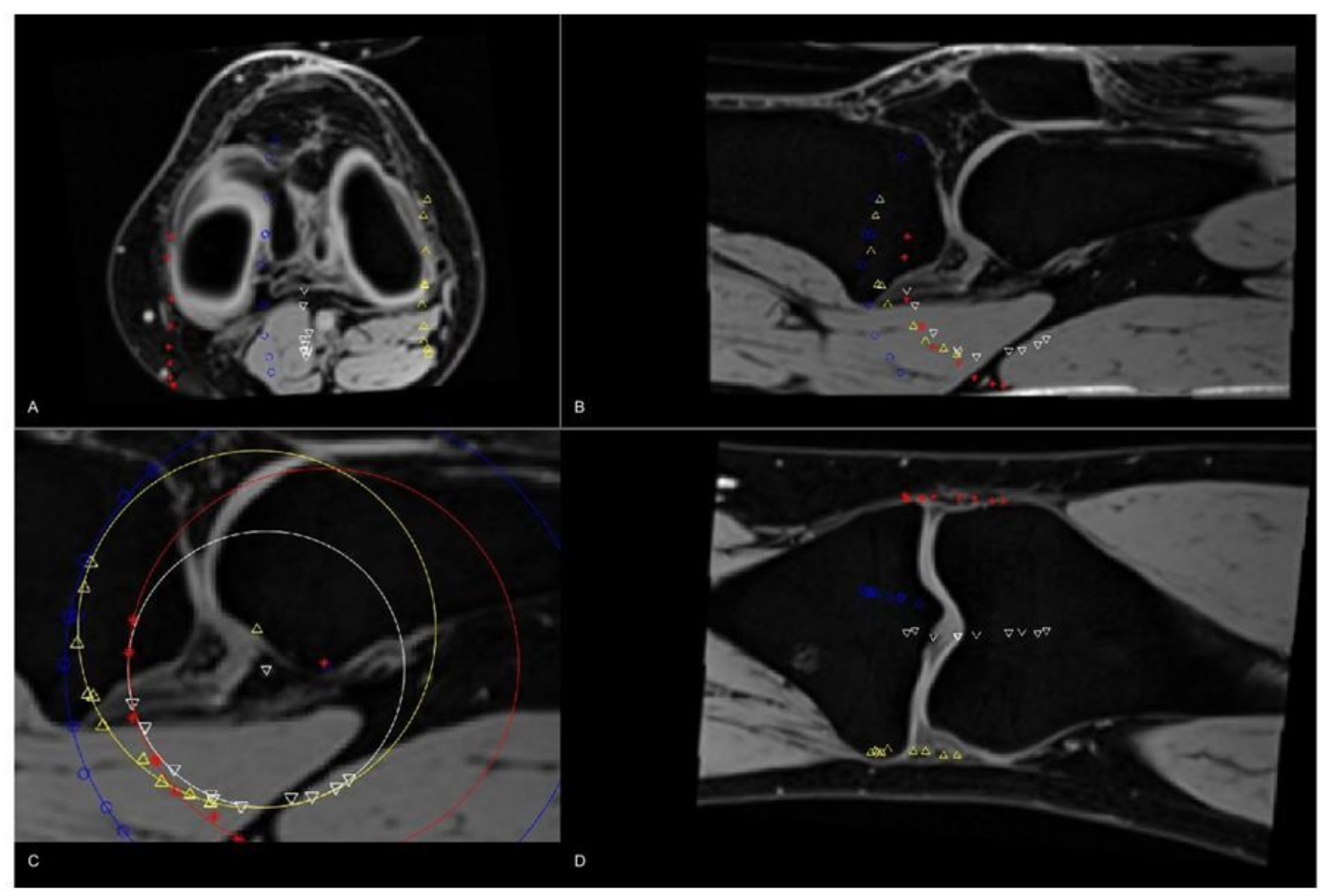

\section{Figure 3}

The VLM0_Tibia after rotation and the projections of the feature points at different angles of view. With the increase in the degree of knee flexion, none of the feature points showed a significant tendency of decrease in the radius of rotation. It seemed that the motion trajectory of these feature points was largely restricted to a circular motion, while being impacted from time to time by the inversion/eversion and internal/external rotation of the tibia. Such impacts were temporary and non-persistent, as the motion trajectory of these feature points would quickly return to the vicinity of the circle. A) A MRI image perpendicular to Z-axis and the projections of the feature points B) A MRI image perpendicular to $\mathrm{Y}$-axis and the projections of the feature points $\mathrm{C}$ ) A locally magnified $\mathrm{Y}$-axis image with the rotation center of each feature point displayed. D) A MRI image perpendicular to X-axis and the projections of the feature points Inverted white triangle: FPtibia1, tibial insertion of posterior cruciate ligament (PCL) Blue circle: FPtibia2, located in the middle of the anterior margin of tibial plateau Yellow triangle: FPtibia3, located in the middle of the lateral margin of tibial plateau Red asterisk: FPtibia4, located in the middle of the medial margin of tibial plateau 


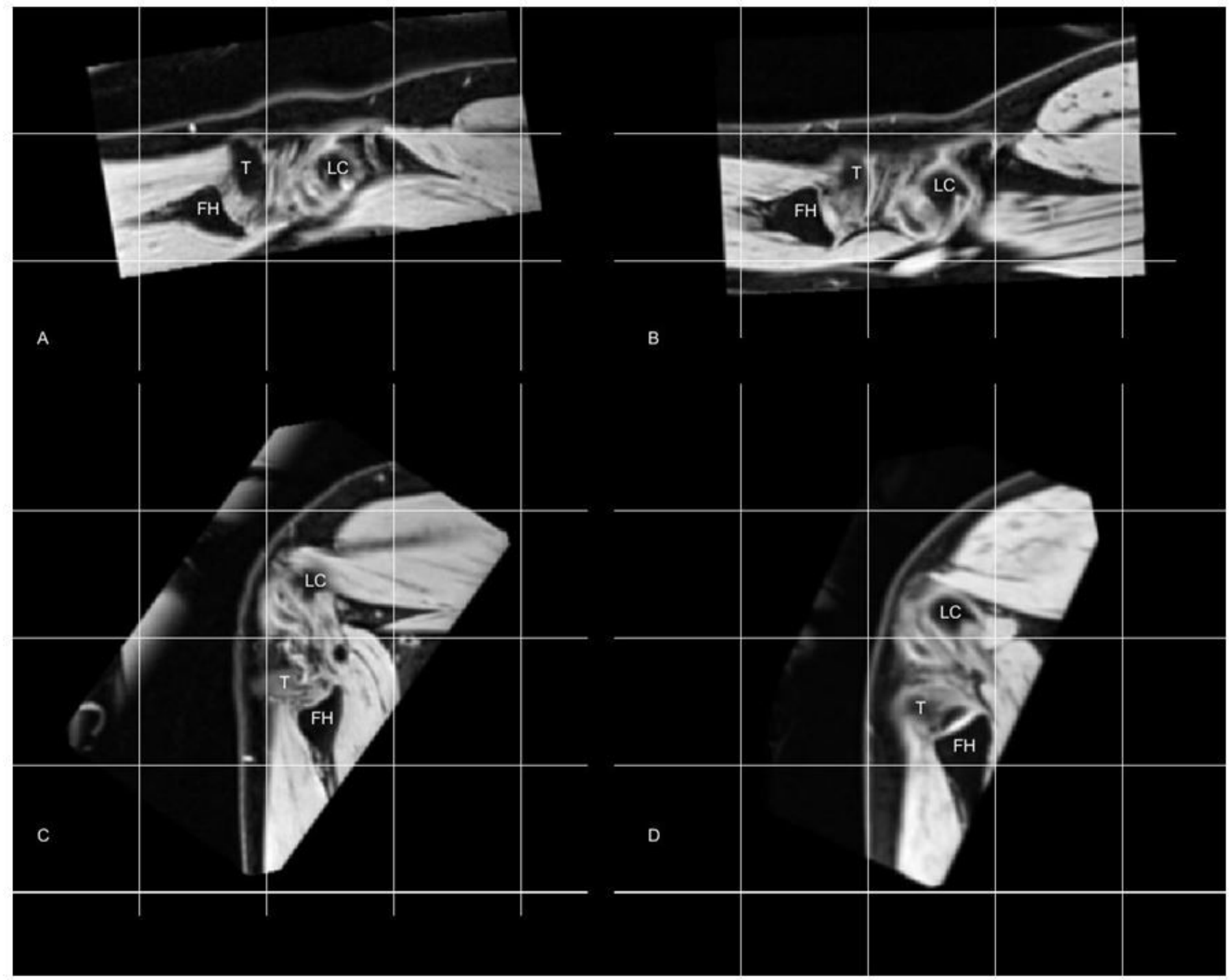

\section{Figure 4}

Another method to determine the direction of the axis of rotation The lateral femoral condyle in slice $A$ and slice $C$ are equal in size, but the tibia in slice $A$ was larger than that in slice $C$, indicating that in the direction of TEA, the tibia moves medially when the knee was flexed, so the rotation axis was not parallel to TEA. The lateral condyle of the femur in slice $B$ and slice $D$ are equal in size, and the tibia in the two slices were also of the same size, which was due to the fact that the rotation axis was parallel with the $Y$ axis. (The direction of femur in VLM0_Femur, VLM90_Femur and VLMO_Tibia were in the same direction). A) Rotate VLM0_Femur (inverted at 2 degrees) so that the TEA is parallel to the $Y$-axis; at this point, this sagittal slice passes through the lateral femoral condyle B) Rotate VLM0_Femur (first inverted at 5 degrees and then medially rotated at 3 degrees) so that the roration axis is parallel to the $\mathrm{Y}$-axis; at this point, this sagittal slice passes through the lateral femoral condyle C) Rotate VLM90_Femur (inverted at 2 degrees) so that the TEA is parallel to the Y-axis; at this point, this sagittal slice passes through the lateral femoral condyle D) Rotate VLM90_Femur (first inverted at 5 degrees and then medially rotated at 3 
degrees) so that the roration axis is parallel to the Y-axis; at this point, this sagittal slice passes through the lateral femoral condyle LC\ Lateral condyle of femur T囚Tibia FH囚Fibular head

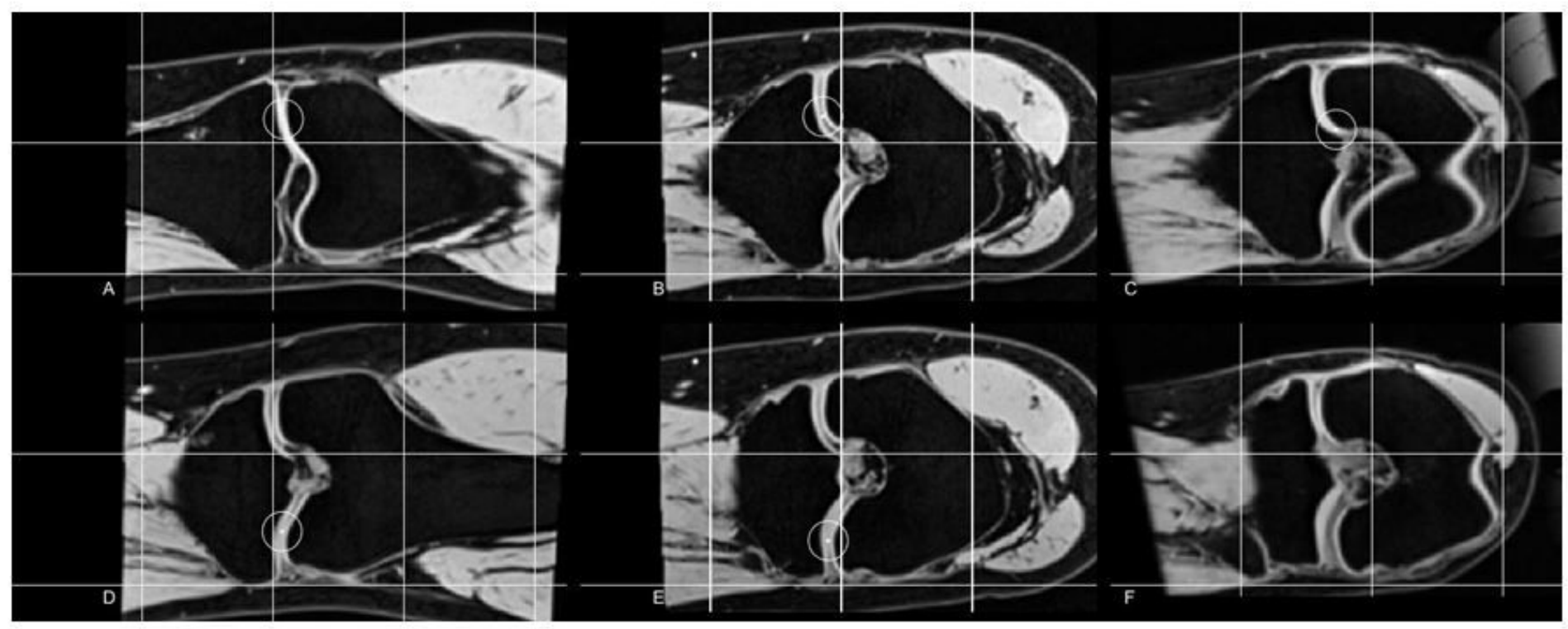

\section{Figure 5}

The greater the flexing angle of the knee, the closer the tibia-femur contact area to the posterolateral side of the tibial plateau. The joint space varies greatly with different knee angles. The circles represent the area closest to the tibial plateau from the medial/lateral femoral condyle A) VLM20_Tibia B) VLM50_Tibia C) VLM80_Tibia D) VLM20_Tibia E) VLM50_Tibia F) VLM80_Tibia, the lateral femoral condyle has been separated from the tibial plateau

\section{Supplementary Files}

This is a list of supplementary files associated with this preprint. Click to download.

- appendixWITHpic.doc 